\title{
Practical and Regioselective Synthesis of C4-Alkylated Pyridines
}

\author{
Jin Choi ${ }^{\dagger}$, Gabriele Laudadio ${ }^{\dagger}$, Edouard Godineau ${ }^{\dagger \dagger}$, Phil S. Baran*† \\ ${ }^{\dagger}$ Department of Chemistry, Scripps Research, 10550 North Torrey Pines Road, La Jolla, California 92037, United States \\ †Process Research, Syngenta Crop Protection, Schaffhauserstrasse 101, CH-4332 Stein, Switzerland
}

\begin{abstract}
The direct position-selective $\mathrm{C}-4$ alkylation of pyridines has been a longstanding challenge in heterocyclic chemistry, particularly from pyridine itself. Historically this has been addressed using pre-functionalized materials to avoid overalkylation and mixtures of regioisomers. This study reports the invention of a simple maleate-derived blocking group for pyridines that enables exquisite control for Minisci-type decarboxylative alkylation at $\mathrm{C}-$ 4 that allows for inexpensive access to these valuable building blocks. The method is employed on a variety of different pyridines and carboxylic acid alkyl donors, is operationally simple, scalable, and is applied to access known structures in a rapid and inexpensive fashion. Finally, this work points to an interesting strategic departure for the use of Minisci chemistry at the earliest possible stage (native pyridine) rather than current dogma that almost exclusively employs Minisci as a late-stage functionalization technique.
\end{abstract}

The power of $\mathrm{C}-\mathrm{H}$ functionalization logic in the context of synthesizing heteroaromatic structures is undeniable. ${ }^{1}$ Its increasing utility in discovery and medicinal chemistry contexts is a testament to its utility in late-stage derivatization enabling structure activity relationships to be rapidly explored. ${ }^{2}$ In particular, the venerable Minisci reaction and its many variants have long been recognized as a way to bypass pre-functionalized heterocycles. ${ }^{3}$ Just as the Friedel-Crafts reaction is commonplace for electron rich arene functionalization via electrophilic substitution, free radicals can react with electron deficient heterocycles by capitalizing on innate reactivity. ${ }^{4}$ In cases where a heterocycle has multiple sites to intercept a free radical, mixtures often result which can be useful in a discovery setting but is problematic when a singular regiochemical outcome is desired (e.g. process scale).$^{5}$ For example, simple 4-alkylated pyridines (1, Figure 1A) are inaccessible using Minisci chemistry if a single regioisomer is desired. In such cases, prefunctionalization is necessary and the logical synthon is halopyridine 2 . This conundrum has rendered the early-stage application of Minisci chemistry on pyridine and mono-substituted pyridines rare in medicinal chemistry and, to our knowledge, non-existent on process scale. A recent collaborative program ${ }^{6}$ within the agrochemical industry brought to our attention the need for a simple and inexpensive solution to this unmet challenge in pyridine alkylation for which available methods were not applicable. To be sure, several attempts to solve this problem from unfunctionalized starting materials have appeared over the past decade (Figure 1B) mostly based on blocking competitive $\mathrm{C}-2$ sites using transient or covalently linked species at the pyridine nitrogen. ${ }^{7}$ Nakao's pioneering studies using bulky Al-based Lewis-acids in an elegant hydroarylation process is limited to olefin donors and must be performed in a glove box. ${ }^{8}$ The Fier group at Merck invented clever oxime-based pyridinium species that could be employed in three examples of $\mathrm{C}-$ $\mathrm{C}$ bond formation with carbon-based nucleophiles. ${ }^{9}$ Finally, the
Hong group reported a radical-type addition using $N$-sulfonamidopyridinium species ${ }^{10}$ using alkyl bromide donors requiring photochemical initiation and super stoichiometric amounts of an expensive silane $\left[(\mathrm{TMS})_{3} \mathrm{SiH}\right] .{ }^{11}$ While this is an important precedent it could not be employed easily on process scale as three steps are needed to install the blocking group (1. $\mathrm{N}$-amination using hydroxylamine- $O$-sulfonic acid, 2. tosylation, and 3. methylation with Meerwein's salt along with 1 column purification, 1 recrystallization). The Buchwald group reported a $\mathrm{Cu}$-catalyzed, selective $\mathrm{C} 4$ functionalization of pyridine with styrenes without a covalent blocking group, via a novel intramolecular rearrangement mechanism mediated by a pyridine coordinated copper species. ${ }^{7 \mathrm{e}, 7 \mathrm{~h}}$ Herein we disclose a highly practical method featuring on a new blocking group based on a simple fumarate backbone (6a) enabling classic Minisci decarboxylative alkylations to take place with exquisite selectivity at $\mathrm{C}-4$ (Figure 1C) under acid-free conditions. Emblematic of this advance is the preparation of 4-cyclohexylpyridine (8) which cannot be accessed from pyridine in a synthetically useful yield and as a mixture of isomers under four different Minisci-type conditions.

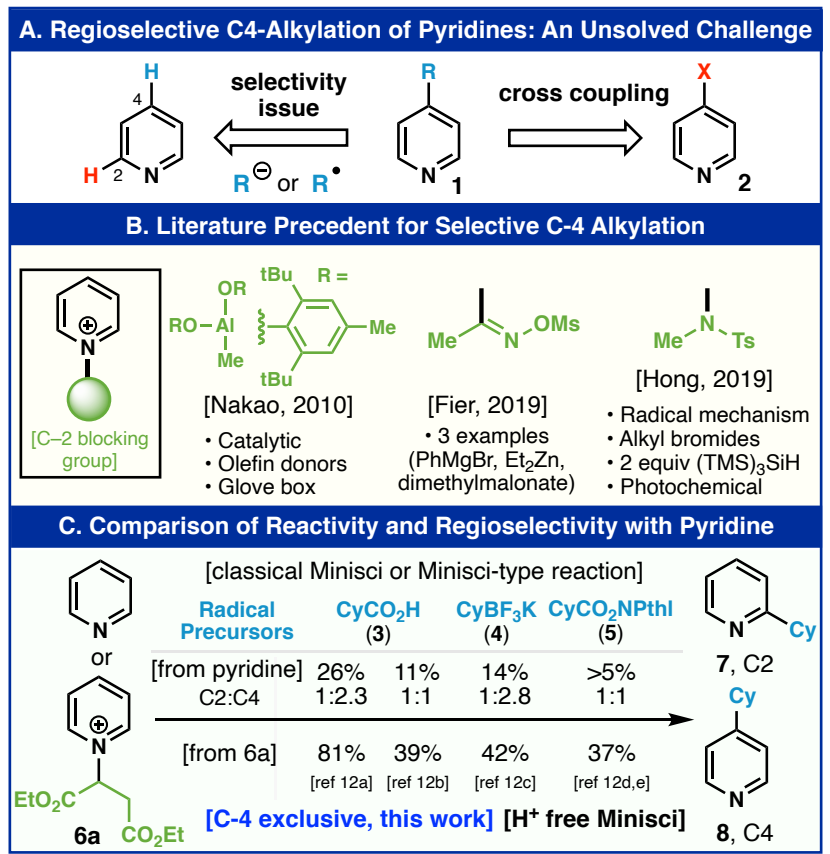

Figure 1. (A) An unsolved challenge in the field of Minisci reaction. (B) Literature precedent. (C) Comparison experiment with pyridine

Tactically, scalable access to valuable structures such as $\mathbf{8}(81 \%$ isolated yield from 6a) can now be enabled with a dramatic reduction in cost. From a strategic perspective, this work opens a new 
dimension of retrosynthetic logic for use of the Minisci transform at an early rather than late stage.

Guided by colleagues at Syngenta (E.G.) several criteria needed to be met for a practical blocking group (BG) design, such as: (1) derivation from feedstock materials (ca. \$5/mole), (2) simple installation and removal, (3) high stability, ease of handling, and solubility in multiple solvents, and most critically, (4) complete regiochemical control to avoid the need for any chromatography. Towards this end, multiple BGs were explored with most falling into one of two categories (Figure 2A): (1) simple BG installation with either modest or low reactivity under Minisci conditions (BG1, 2, 4,6 ) or (2) difficulty in forming a stable BG adduct. After extensive exploration, BG10 emerged as an ideal candidate satisfying all of the criteria laid out above. BG9 was the only other moderately successful one however it exhibited reduced reactivity towards Minisci addition. The preparation of pyridinium $\mathbf{6 a}$ with BG10 could be prepared through a simple, chromatography-free, two-step sequence starting from commodity materials (pyridine and maleic acid) followed by esterification. The structure of pyridinium $\mathbf{6 a}$ was confirmed by X-ray crystallography and contained the ethyl sulfonate as a counter-anion. This crystalline salt represents a straightforward gateway to a variety of $\mathrm{C}-4$ alkylated pyridines (vide infra) and has been commercialized by Sigma-Aldrich (catalog \# ALD00619).

\section{A. A Fumarate-Derived Blocking Group For Minisci: Discovery}

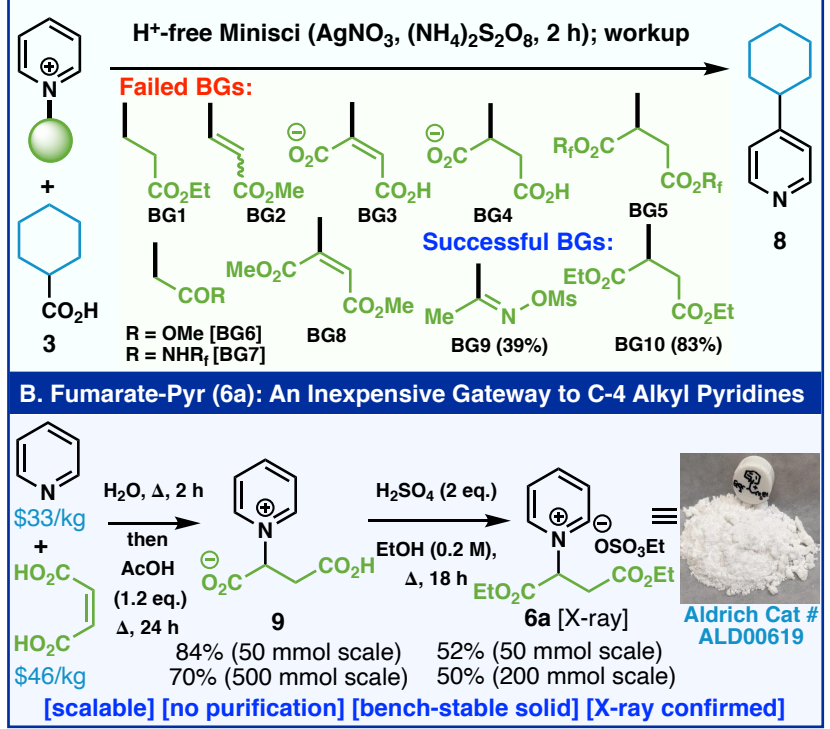

Figure 2. (A) Fumarate-derived blocking group for Minisci reaction in discovery stage (B) The pyridinium $\mathbf{6 a}$ as an inexpensive gateway to $\mathrm{C} 4$ alkylated pyridine synthesis.

The generalization of this fumarate-based BG approach is illustrated in Table 1 using acid-free Minisci conditions on a range of primary, secondary, and tertiary carboxylic acids. Although these C -4 alkylated pyridines appear simple, it is instructive to comment on the means by which such compounds were previously prepared. In nearly all cases the current method represents a more practical and cost-effective solution. In the case of primary carboxylic acid adducts, pyridine $\mathbf{1 1}$ was accessed from prefunctionalized 4methylpyridine via lithiation- $\mathrm{S}_{\mathrm{N}} 2$ with corresponding alkyl bromide $\left(c a . \$ 105 / \mathrm{g}^{13 b}\right) .{ }^{14}$ Pyridine 12 was obtained through an analogous sequence using an alkyl bromide containing a protected alcohol requiring subsequent deprotection and chlorination ( $\mathrm{ca}$. $\left.\$ 945 / \mathrm{g}^{13 \mathrm{~b}}\right) .{ }^{15}$ Pyridine 13, 14 and $\mathbf{1 7}$ were previously prepared via photochemical addition on 4-vinyl pyridine. ${ }^{16}$ Pyridine 15 required a Pd-cross coupling on either 4-vinyl or 4-bromopyridine (Heck ${ }^{17 a}$ or Sonogashira, ${ }^{17 \mathrm{~b}}$ respectively) followed by reduction ( $c a$. $\$ 530 / \mathrm{g}^{13 \mathrm{c}}$ ). Similarly, pyridine $\mathbf{1 6}$ can be accessed via reduction of the Heck product of 4-vinylpyridine and an aryl iodide. ${ }^{18}$

Numerous secondary carboxylic acids were employed to access such pyridines with high simplicity when placed in context. For example, pyridine $\mathbf{8}$ has been prepared multiple times leading either to mixtures (e.g., Figure 2B) or requiring pre-functionalized pyridines $\left(c a . \$ 584 / \mathrm{g}^{13 \mathrm{~d}}\right) .{ }^{19}$ Similarly, pyridine 18 has been accessed from 4-bromopyridine through photochemical and electrochemical reductive couplings or by employing Hong's BG (Figure 1B) and a Hantzsch ester radical precursor ${ }^{10 \mathrm{~b}}\left(\mathrm{ca} . \$ 150 / \mathrm{g}^{13 \mathrm{e}}\right)$. Pyridine 21 has been accessed either via cross coupling/Hydrogenation ${ }^{20 \mathrm{a}}$ or C4-selective Grignard addition using TBSOTf to generate a transient BG and reoxidation ${ }^{20 \mathrm{~b}}\left(\mathrm{ca} . \$ 100 / \mathrm{g}^{13 \mathrm{~b}}\right)$. Cyclopropyl containing pyridine 23 was accessed either from 4-bromo or 4-Bpin pyridine via Suzuki or Grignard addition/rearomatization $\left(\mathrm{ca} . \$ 226 / \mathrm{g}^{13 \mathrm{a}}\right){ }^{21}$ The trivial cyclohexanone pyridine $\mathbf{2 5}$ has only been accessed in a controlled fashion using multistep routes with protecting groups and FG manipulations ( $c$ a. $\left.\$ 871 / \mathrm{g}^{13 f}\right){ }^{22}$

Many of the quaternary center containing C-4 alkylated pyridines (derived from tertiary carboxylic acids) prepared here are new (29-33) and are likely desirable starting materials for medicinal chemistry programs. Of the known alkylated pyridines in this series, two were prepared as mixtures of regioisomers using radical chemistry (26 and $\mathbf{2 8})^{23,24}$ or via Minisci addition to 4-cyanopyridine. $^{25}$

The chemistry outlined above is not limited to the parent pyridine 6a but can also be employed on mono $(\mathbf{6 b}, \mathbf{6 d - i})$ or bis $(\mathbf{6 c})$ substituted pyridines. Pyridines 35, 39, and $\mathbf{4 1}$ are new compounds and might be challenging to access controllably from the parent pyridines in other ways. Pyridines such as 37, 38 (ca. $\$ 1620 / \mathrm{g}^{13 \mathrm{~g}}$ ), and 40 have previously been synthesized either through Grignard addition/oxidation sequences ${ }^{7 \mathrm{c}}$ or via Hong's HAT-based method $^{10 \mathrm{~d}}$ employing BG's similar to that in Figure 1B.

It is worth noting that pyridines $\mathbf{8 , 1 2}, \mathbf{2 7}$ and $\mathbf{2 8}$ have been prepared on a gram-scale with no significant reduction in yield. The limitations of this reaction (see SI for full disclosure) stem from the acidic conditions used to install the $\mathrm{BG}$ and a lack of tolerance for preexisting $\mathrm{C}-2$ functionality.

Having facile access to pure $\mathrm{C}-4$ alkylated pyridines open up a new opportunity for early-stage Minisci chemistry to be employed in the synthesis of 2,4-difunctionalized systems. Historically, such heterocycles are prepared by employing Minisci at the end of a sequence in order to obtain more regioselective outcomes. As shown in Figure 3A, a reversal of this traditional choreography is now feasible. Thus, adduct 8 can be submitted to known $\mathrm{C}-2$ selective pyridine functionalizations such as carbamoylation ${ }^{26}$, cyanation ${ }^{9}$, and amidation $^{27}$ to afford pyridines $\mathbf{4 2 , 4 3}$, and $\mathbf{4 4}$, respectively. This sequence of events is general and can be utilized to obtain $\mathbf{4 6}$ (Minisci followed by borono-Minisci ${ }^{28}$ ), 47 and 48 (double Minisci), and 49 (Minisci followed by amidation). Conventional retrosynthesis of such compounds would likely involve pre-functionalized handles for controlling regiochemistry whereas in the present case innate reactivity and the fumarate-BG overcomes this challenge.

As mentioned above (Table 1), many of the pyridines reported herein have been prepared by less direct pathways and this is graphically depicted for pyridines 12, 27, and 25 in Figure 3B. The avoidance of pre-functionalized pyridines, pyrophoric reagents, and expensive transition metals are highlights of this method. Moreover, a proof of concept is shown for how the fungicide (oomycetes) candidate 54 could conceivably be accessed in a far more practical way 
Reaction Scope: Regioselective C4-Alkylation of Pyridines

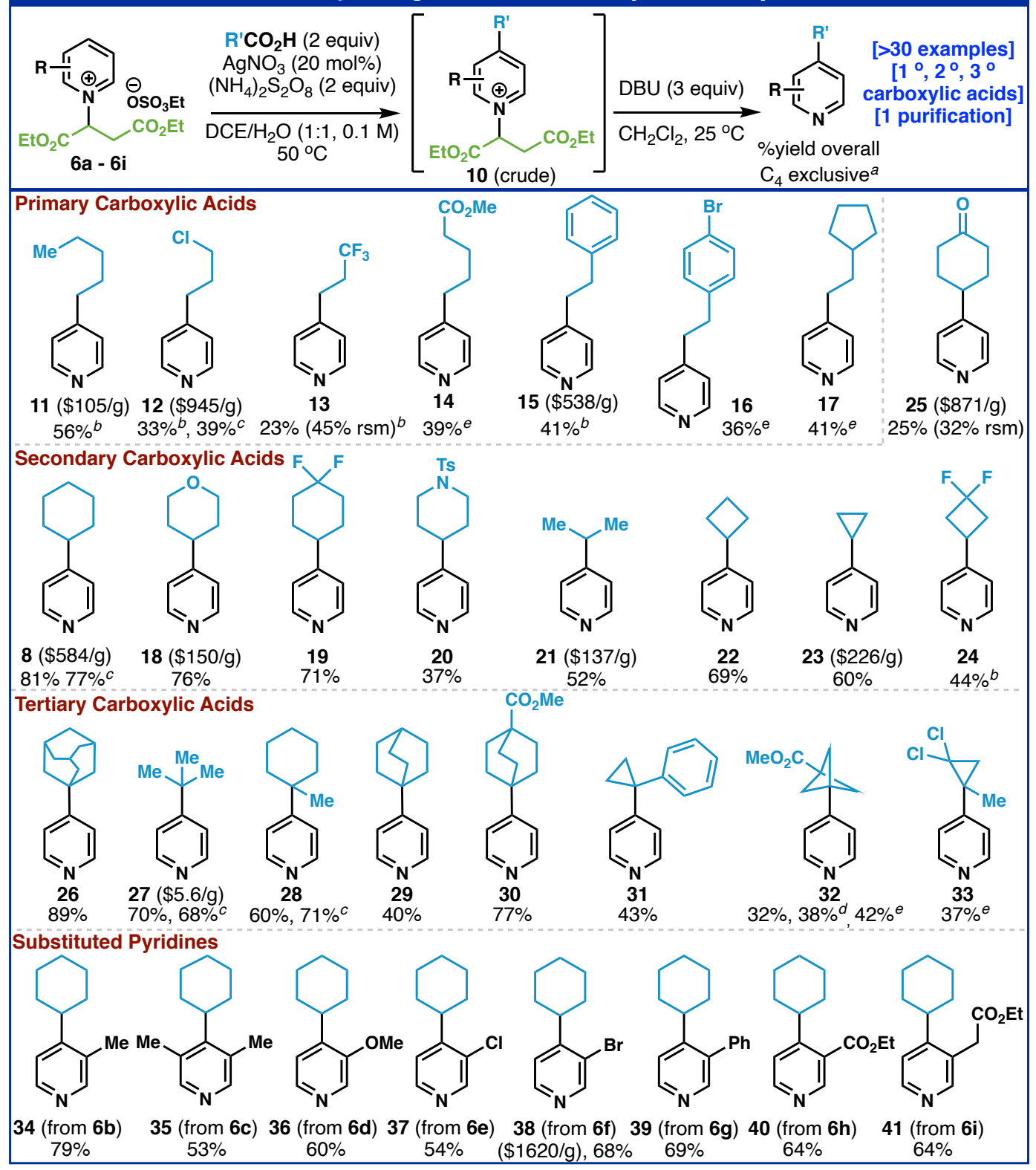

Table 1. Reaction scope of the regioselective Minisci reaction. a) 6a $(0.5 \mathrm{mmol})$, carboxylic acid $(1.0 \mathrm{mmol}), \mathrm{AgNO}_{3}\left(20 \mathrm{~mol}^{\circ}\right)$, $\left(\mathrm{NH}_{4}\right)_{2} \mathrm{~S}_{2} \mathrm{O}_{8}(1.0 \mathrm{mmol})$, DCE: $\mathrm{H}_{2} \mathrm{O}=1: 1,0.1 \mathrm{M}, 50{ }^{\circ} \mathrm{C}, 2 \mathrm{~h}$. The regioselectivity was determined by crude NMR after first step and confirmed again after final purification step. b) using carboxylic acid ( $2.0 \mathrm{mmol}, 4$ equiv) on the Minisci reaction step and DBU (3.0 mmol, 6 equiv) on the removal step. c) $5.0 \mathrm{mmol}$ scale reaction. d) carboxylic acid was used as a limiting reagent. e) performed in 0.3 M. See Supporting Information for detailed experimental procedures.

from pyridine. Prior studies employed chemistry that was cost-prohibitive for the agrochemical industry commencing from $\mathbf{5 1}$ and employing expensive boronate ester 52, N-oxide chemistry, toxic TMSCN, and a Pd catalyst to access 1,3-disubstituted $\mathbf{5 3}$ which required a subsequent hydrogenation to remove the 3,4-unsaturation. ${ }^{29}$ In contrast, the two-stage Minisci approach from $6 \mathbf{a}$ accesses a synthetically equivalent intermediate $\mathbf{5 0}$ directly without any of those drawbacks. Finally, as a demonstration of practicality in both medicinal and process scenarios pyridine $\mathbf{2 6}$ can be prepared and purified either through column chromatography or through a simple extraction/washing protocol.
To summarize, a simple solution to the longstanding challenge of practical $\mathrm{C}-4$ alkylation of pyridines has been presented using a simple blocking group derived from inexpensive maleic acid. The resulting pyridinium species is stable and, in many instances, crystalline. The resulting functionalization can be accomplished using classic Minisci conditions without the addition of any acid and proceeds to give a singular adduct at $\mathrm{C}-4$. The scope of this reaction is broad and can be strategically used in concert with other functionalizations or as a stand-alone method to provide high value pyridines that despite their trivial appearance, have posed challenges for direct and inexpensive synthesis in a scalable way. 


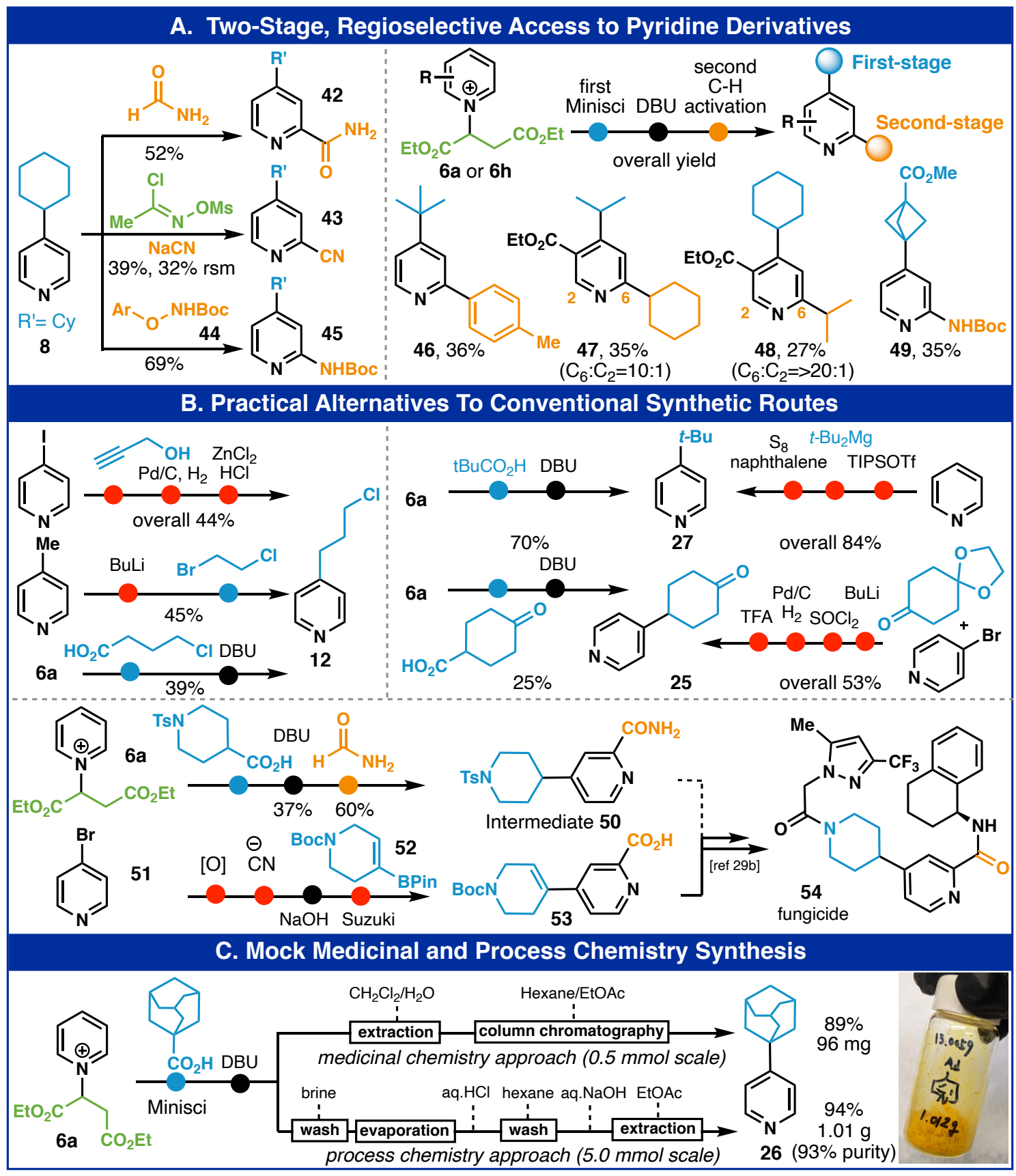

Figure 3. (A) Two-stage derivatization. (B) Practical alternatives to conventional synthetic routes. (C) Mock medicinal and process chemistry synthesis. See Supporting Information for detailed experimental procedures.

\section{AUTHOR INFORMATION}

\section{Corresponding Author}

pbaran@scripps.edu

\section{ORCID}

Jin Choi: 0000-0001-5759-8419, Gabriele Laudadio: 0000-00022749-8393 Edouard Godineau: 0000-0002-5958-4317, Phil S. Baran: 0000-0001-9193-905

\section{Notes}

The authors declare no competing financial interest.

\section{ACKNOWLEDGMENT}

Financial support for this work was provided the NIH (GM118176) and Syngenta Crop Protection. We are thankful to the Vivozon Inc. (Young Scientist Grant and Postdoctoral Fellowship, J.C.) and the George E. Hewitt Foundation (G.L.). We are grateful to Dr. D.-H. Huang and Dr. L. Pasternack (Scripps Research) for NMR spectroscopic assistance, to Dr. J. Chen, B. Sanchez, E. Sturgell (Scripps Research Automated Synthesis Facility), Dr. G. Siuzdak and E. Billings (Scripps Research, The Center of Metabolomics and Mass Spectrometry) for assistance with HRMS, to Dr. M. Gembicky (UCSD) for X-ray crystallographic analysis. 


\section{REFERENCES}

1) (a) Yu, J.-Q.; Shi, Z., CH activation. Springer: 2010; Vol. 292. (b) Brückl, T.; Baxter, R. D.; Ishihara, Y.; Baran, P. S., Innate and Guided C-H Functionalization Logic. Acc. Chem. Res. 2012, 45, 826-839. (c) Murakami, K.; Yamada, S.; Kaneda, T.; Itami, K., C-H Functionalization of Azines. Chem. Rev. 2017, 117, 9302-9332.

2) (a) Brow, D. G.; Bostrom, J., Analysis of Past and Present Synthetic Methodologies on Medicinal Chemistry: Where Have All the New Reactions Gone? J. Med. Chem. 2016, 59, 4443-4458. (b) Cernak, T.; Dykstra, K. D.; Tyagarajan, S.; Vachal, P.; Krska, S. W., The Medicinal Chemist's Toolbox for Late-stage Functionalization of Drug-like Molecules. Chem. Soc. Rev. 2016, 45, 546-576. (c) Bostrom, J.; Brown, D. G.; Young, R. J.; Keseru, G. M., Expanding the Medicinal Chemistry Synthetic Toolbox. Nat. Rev. Drug. Discov. 2018, 17, 709-727.

3) For reviews of Minisci reaction see; (a) Minisci, F.; Galli, R.; Cecere, M.; Malatesta, V.; Caronna, T., Nucleophilic Character of Alkyl Radicals: New Syntheses by Alkyl Radicals Generated in Redox Processes. Tetrahedron Lett. 1968, 9, 5609-5612. (b) Duncton, M. A. J., Minisci reactions: Versatile $\mathrm{CH}$-functionalizations for Medicinal Chemists. Med. Chem. Comm. 2011, 2, 1135-1161. (c) Proctor, R. S. J.; Phipps, R. J., Recent Advances in Minisci-Type Reactions. Angew. Chem. Int. Ed. 2019, 58, 13666-13699. (d) Wang, W. G.; Wang, S. F., Recent Advances in Minisci-type Reactions and Applications in Organic Synthesis. Cur. Org. Chem. 2021, 25, 894-934.

4) Ishihara, Y.; Montero, A.; Baran, P. S. The Portable Chemist's Consultant: A Survival Guide for Discovery, Process, and Radiolabeling. Macintosh Publishing, 2013. (electronic book) https://books.apple.com/us/book/the-portable-chemists-consultant/id618463142 (accessed May 3, 2021).

5) O'Hara, F.; Blackmond, D. G.; Baran, P. S., Radical-Based Regioselective $\mathrm{C}-\mathrm{H}$ Functionalization of Electron-Deficient Heteroarenes: Scope, Tunability, and Predictability. J. Am. Chem. Soc. 2013, 135, 12122-12134.

6) Michaudel, Q.; Ishihara, Y.; Baran, P. S., Academia-Industry Symbiosis in Organic Chemistry. Acc. Chem. Res. 2015, 48, 712-721.

7) (a) Corey, E. J.; Tian, Y., Selective 4-Arylation of Pyridines by a Nonmetalloorganic Process. Org. Lett. 2005, 7, 5535-5537. (b) Tsai, C. C.; Shih, W. C.; Fang, C. H.; Li, C. Y.; Ong, T. G.; Yap, G. P. A., Bimetallic Nickel Aluminun Mediated Para-Selective Alkenylation of Pyridine: Direct Observation of $\eta^{2}, \eta^{1}$-Pyridine $\mathrm{Ni}(0)$ - $\mathrm{Al}$ (III) Intermediates Prior to CH Bond Activation. J. Am. Chem. Soc. 2010, 132, 11887-11889. (c) Chen, Q.; du Jourdin, X. M.; Knochel, P., Transition-Metal-Free BF 3 -Mediated Regioselective Direct Alkylation and Arylation of Functionalized Pyridines Using Grignard or Organozinc Reagents. J. Am. Chem. Soc. 2013 135, 4958-4961. (d) Ma, X.; Dang, H.; Rose, J. A.; Rablen, P.; Herzon, S. B., Hydroheteroarylation of Unactivated Alkenes Using $N$-Methoxyheteroarenium Salts. J. Am. Chem. Soc. 2017, 139, 5998-6007. (e) Gribble, M. W.; Guo, S.; Buchwald, S. L., Asymmetric Cu-Catalyzed 1,4Dearomatization of Pyridines and Pyridazines without Preactivation of the Heterocycle or Nucleophile. J. Am. Chem. Soc. 2018, 140, 5057-5060. (f) Zhang, W.-B.; Yang, X.-T.; Ma, J.-B.; Su, Z.-M.; Shi, S.-L., Regioand Enantioselective C-H Cyclization of Pyridines with Alkenes Enabled by a Nickel $/ N$-Heterocyclic Carbene Catalysis. J. Am. Chem. Soc. 2019, 141, 5628-5634. (g) Wang, Y.; Li, R.; Guan, W.; Li, Y.; Li, X.; Yin, J.; Zhang, G.; Zhang, Q.; Xiong, T.; Zhang, Q., Organoborohydride-catalyzed Chichibabin-type C4-position alkylation of pyridines with alkenes assisted by organoboranes. Chem. Sci. 2020, 11, 11554-11561. (h) Gribble, M. W.; Liu, R. Y.; Buchwald, S. L., Evidence for Simultaneous Dearomatization of Two Aromatic Rings under Mild Conditions in $\mathrm{Cu}(\mathrm{I})$ Catalyzed Direct Asymmetric Dearomatization of Pyridine. J. Am. Chem. Soc. 2020, 142, 11252-11269. (i) Obradors, C.; List, B., Azine Activation via Silylium Catalysis. J. Am. Chem. Soc. 2021, 143, 6817-6822.

For representative review, see: (j) Bull, J. A.; Mousseau, J. J.; Pelletier, G.; Charette, A. B., Synthesis of Pyridine and Dihydropyridine Derivatives by Regio- and Stereoselective Addition to $N$-Activated Pyridines. Chem. Rev. 2012, 112, 2642-2713. (k) Rossler, S. L.; Jelier, B. J.; Magnier, E.; Dagousset, G.; Carreira, E. M.; Togni, A., Pyridinium Salts as Redox-Active Functional Group Transfer Reagents. Angew. Chem. Int Ed. 2020, 59, 9264-9280. (1) Zhou, F.-Y.; Jiao, L., Recent Developments in Transition-Metal-Free Functionalization and Derivatization Reactions of Pyridines. Synlett 2021, 32, 159-178.

8) Nakao, Y.; Yamada, Y.; Kashihara, N.; Hiyama, T., Selective C-4 Alkylation of Pyridine by Nickel/Lewis Acid Catalysis. J. Am. Chem. Soc. 2010, 132, 13666-13668.
9) Fier, P. S., A Bifunctional Reagent Designed for the Mild, Nucleophilic Functionalization of Pyridines. J. Am. Chem. Soc. 2017, 139, 94999502.

10) (a) Moon, Y.; Park, B.; Kim, I.; Kang, G.; Shin, S.; Kang, D.; Baik, M. H.; Hong, S., Visible Light Induced Alkene Aminopyridylation using $\mathrm{N}$-aminopyridinium Salts as Bifunctional Reagents. Nat. Commun. 2019 , 10, 4117. (b) Kim, I.; Park, S.; Hong, S., Functionalization of Pyridinium Derivatives with 1,4-Dihydropyridines Enabled by Photoinduced Charge Transfer. Org. Lett. 2020, 22, 8730-8734. (c) Shin, S.; Lee, S.; Choi, W. Kim, N.; Hong, S., Visible-Light-Induced 1,3-Aminopyridylation of [1.1.1] Propellane with $N$-Aminopyridinium Salts. Angew. Chem. Int. Ed. 2021, 60, 7873-7879. (d) Lee, W.; Jung, S.; Kim, M.; Hong, S., Site-Selective Direct C-H Pyridylation of Unactivated Alkanes by Triplet Excited Anthraquinone. J. Am. Chem. Soc. 2021, 143, 3003-3012. (e) Kim, M.; You, E.; Park, S.; Hong, S., Divergent Reactivity of Sulfinates with Pyridinium Salts Based on One- versus Two-electron Pathways. Chem. Sci., 2021, 12, 6629-6637.

11) Jung, S.; Shin, S.; Park, S.; Hong, S., Visible-Light-Driven C4-Selective Alkylation of Pyridinium Derivatives with Alkyl Bromides. J. Am. Chem. Soc. 2020, 142, 11370-11375.

12) (a) Minisci, F.; Bernardi, R.; Bertini, F.; Galli, R.; Perchinummo, M., Nucleophilic character of alkyl radicals-VI: A New Convenient Selective Alkylation of Heteroaromatic Bases. Tetrahedron 1971, 27, 35753579. (b) Galloway, J. D.; Mai, D. N.; Baxter, R. D., Silver-Catalyzed Minisci Reactions Using Selectfluor as a Mild Oxidant. Org. Lett. 2017, 19, 5772-5775. (c) Presset, M.; Fleury-Brégeot, N.; Oehlrich, D.; Rombouts, F.; Molander, G. A., Synthesis and Minisci Reactions of Organotrifluoroborato Building Blocks. J. Org. Chem. 2013, 78, 4615-4619. (d) Liu, Y.; Xue, L.; Shi, B.; Bu, F.; Wang, D.; Lu, L.; Shi, R.; Lei, A., Catalyst-free electrochemical decarboxylative cross-coupling of $\mathrm{N}$-hydroxyphthalimide esters and $N$-heteroarenes towards $\mathrm{C}\left(\mathrm{sp}^{3}\right)-\mathrm{C}\left(\mathrm{sp}^{2}\right)$ bond formation. Chem. Commun. 2019, 55, 14922-14925. (e) Niu, K.; Song, L.; Hao, Y.; Liu, Y.; Wang, Q., Electrochemical decarboxylative C3 alkylation of quinoxalin-2(1H)-ones with $N$-hydroxyphthalimide esters. Chem. Commun. 2020, 56, 11673-11676.

13) Reagent price based on (a) Sigma-Aldrich (b) Combi-Blocks (c) BLD Pharm (d) Enamine (e) Oakwood Chemical (f) AstaTech (g) Aurum Pharmtech (accessed April $8^{\text {th }}, 2021$ )

14) Howell, J. M.; Feng, K.; Clark, J. R.; Trzepkowski, L. J.; White, M. C., Remote Oxidation of Aliphatic C-H Bonds in Nitrogen-Containing Molecules. J. Am. Chem. Soc. 2015, 137, 14590-14593.

15) (a) Kassiou, M.; Read, R. W.; Shi, X.-Q., Synthesis and Evaluation of Halogenated Dibenzodiazepines as Muscarinic Receptor Ligands. Bioorg. Med. Chem. Lett. 1997, 7, 799-804. (b) Hoang, V.-H.; Tran, P.T.; Cui, M.; Ngo, V. T. H.; Ann, J.; Park, J.; Lee, J.; Choi, K.; Cho, H.; Kim, H.; Ha, H.-J.; Hong, H.-S.; Choi, S.; Kim, Y.-H.; Lee, J., Discovery of Potent Human Glutaminyl Cyclase Inhibitors as Anti-Alzheimer's Agents Based on Rational Design. J. Med. Chem. 2017, 60, 2573-2590.

16) (a) Straathof, N. J. W.; Cramer, S. E.; Hessel, V.; Noel, T., Practical Photocatalytic Trifluoromethylation and Hydrotrifluoromethylation of Styrenes in Batch and Flow. Angew. Chem. Int. Ed. 2016, 55, 1554915553. (b) Wang, Y. W.; Deng, L. F.; Zhang, X.; Mou, Z. D.; Niu, D. W., A Radical Approach to Making Unnatural Amino Acids: Conversion of $\mathrm{C}-\mathrm{S}$ Bonds in Cysteine Derivatives into C-C bonds. Angew. Chem. Int. Ed. 2021, 60, 2155-2159. (c) Quan, Y.; Song, Y.; Shi, W.; Xu, Z.; Chen, J. S.; Jiang, X.; Wang, C.; Lin, W., Metal-Organic Framework with Dual Active Sites in Engineered Mesopores for Bioinspired Synergistic Catalysis. J. Am. Chem. Soc. 2020, 142, 8602-8607.

17) (a) Efange, S. M. N.; Michelson, R. H.; Remmel, R. P.; Boudreau, R. J.; Dutta, A. K.; Freshler, A., Flexible $N$-methyl-4-phenyl-1,2,3,6-tetrahydropyridine Analog: Synthesis and Monoamine Oxidase Catalyzed Bioactivation. J. Med. Chem. 1990, 33, 3133-3138. (b) Takhi, M.; Hosahalli, S.; Panigrahi, S. K.; Mahadari, M. K.; Kottam, S. R.; Abd Rahman, N.; Yusof, R. Substituted Pyridine Derivatives as FABI Inhibitors, PCT WO 201380222 A1 June 6, 2013

18) Kalashnikov, V. V.; Tomilova, L. G., Catalytic Reduction of an $\alpha, \beta-$ Disubstituted Alkene with Sodium Borohydride in the Presence of Tetratert-butylphthalocyanine Complexes. Mendeleev Commun. 2007, 17, 343-344.

19) (a) Minisci, F.; Fontana, F., Mechanism of the Gif-Barton Type Alkane Functionalization by Halide and Pseudohalide Ions. Tetrahedron Lett. 1994, 35, 1427-1430. (b) For selected examples from pre-functionalized pyridines see: Molander, G. A.; Argintaru, O. A.; Aron, I.; Dreher, S. D., Nickel-Catalyzed Cross-Coupling of Potassium Aryl- and Heteroaryltrifluoroborates with Unactivated Alkyl Halides. Org. Lett. 2010, 
12, 5783-5785. (c) Basch, C. H.; Liao, J.; Xu, J.; Piane, J. J.; Watson, M. P., Harnessing Alkyl Amines as Electrophiles for Nickel-Catalyzed Cross Couplings via C-N Bond Activation. J. Am. Chem. Soc. 2017, 139, 53135316. (d) Perry, I. B.; Brewer, T. F.; Sarver, P. J.; Schultz, D. M.; DiRocco, D. A.; MacMillan, D. W. C., Direct Arylation of Strong Aliphatic $\mathrm{C}-\mathrm{H}$ bonds. Nature 2018, 560, 70-75.

20) (a) Friedfeld, M. R.; Margulieux, G. W.; Schaefer, B. A.; Chirik, P. J., Bis(phosphine)cobalt Dialkyl Complexes for Directed Catalytic Alkene Hydrogenation. J. Am. Chem. Soc. 2014, 136, 13178-13181. (b) Akiba, K.; Iseki, Y.; Wada, M., A Convenient Method for the Regioselective Synthesis of 4-Alkyl(aryl)pyridines using Pyridinium Salts. Bull. Chem. Soc. Jpn. 1984, 57, 1994-1999.

21) (a) Lewis, R. T.; Jones, P.; Petrocchi, A.; Reyna, N.; Hamilton, M.; Cross, J.; Tremblay, M.; Leonard, P. G. Compounds, PCT WO 2018 136887 July 26, 2018. (b) Panda, S.; Coffin, A.; Nguyen, Q. N.; Tantillo, D. J.; Ready, J. M., Synthesis and Utility of Dihydropyridine Boronic Esters. Angew. Chem. Int. Ed. 2016, 55, 2205-2209.

22) Zhou, J.; Jiang, Q.; Fu, P.; Liu, S.; Zhang, S.; Xu, S.; Zhang, Q., Syntheses of 4-(Heteroaryl)cyclohexanones via Palladium-Catalyzed Ester $\alpha$-Arylation and Decarboxylation. J. Org. Chem. 2017, 82, 9851-9858. and reference therein.

23) (a) Barton, D. H. R.; Bévière, S. D.; Chavasiri, W., The Functionalization of Saturated Hydrocarbons. Part 25. Ionic Substitution Reactions in GoAgg ${ }^{\mathrm{IV}}$ Chemistry: the Formation of Carbon-halogen Bonds. Tetrahedron 1994, 50, 31-46. (b) Barniol-Xicota, M.; Gazzarrini, S.; Torres, E.; Hu, Y.; Wang, J.; Naesens, L.; Moroni, A.; Vázquez, S., Slow but Steady Wins the Race: Dissimilarities among New Dual Inhibitors of the
Wild-Type and the V27A Mutant M2 Channels of Influenza A Virus. $J$. Med. Chem. 2017, 60, 3727-3738.

24) Pitre, S. P.; Muuronen, M.; Fishman, D. A.; Overman, L. E., Tertiary Alcohols as Radical Precursors for the Introduction of Tertiary Substituents into Heteroarenes. ACS Catal. 2019, 9, 3413-3418.

25) (a) Gao, L.; Wang, G.; Cao, J.; Chen, H.; Gu, Y.; Liu, X.; Cheng, X.; Ma, J.; Li, S., Lewis Acid-Catalyzed Selective Reductive Decarboxylative Pyridylation of $\mathrm{N}$-Hydroxyphthalimide Esters: Synthesis of Congested Pyridine-Substituted Quaternary Carbons. ACS Catal. 2019, 9, 10142-10151.

26) Han, W.; Jin, F.; Zhao, Q.; Du, H.; Yao, L., Acid-Free Silver-Catalyzed Cross-Dehydrogenative Carbamoylation of Pyridines with Formamides. Synlett 2016, 27, 1854-1859.

27) Fier, P. S.; Kim, S.; Cohen, R. D., A Multifunctional Reagent Designed for the Site-Selective Amination of Pyridines. J. Am. Chem. Soc. 2020, 142, 8614-8618.

28) Seiple, I. B.; Su, S.; Rodriguez, R. A.; Gianatassio, R.; Fujiwara, Y.; Sobel, A. L.; Baran, P. S., Direct C-H Arylation of Electron-Deficient Heterocycles with Arylboronic Acids. J. Am. Chem. Soc. 2010, 132, $13194-13196$

29) (a) Sulzer-Mosse, S.; Cederbaum, F.; Lamberth, C.; Berthon, G.; Umarye, J.; Grasso, V.; Schlereth, A.; Blum, M.; Waldmeier, R., Synthesis and fungicidal activity of $N$-thiazol-4-yl-salicylamides, a new family of anti-oomycete compounds. Bioorg. Med. Chem. 2015, 23, 2129-2138. (b) Lamberth, C., Episodes from the Continuous Search for Solutions against Downy Mildew Diseases. Chimia 2019, 73, 571-580. 


\section{Practical and Regioselective Synthesis of C-4 Alkylated Pyridines}

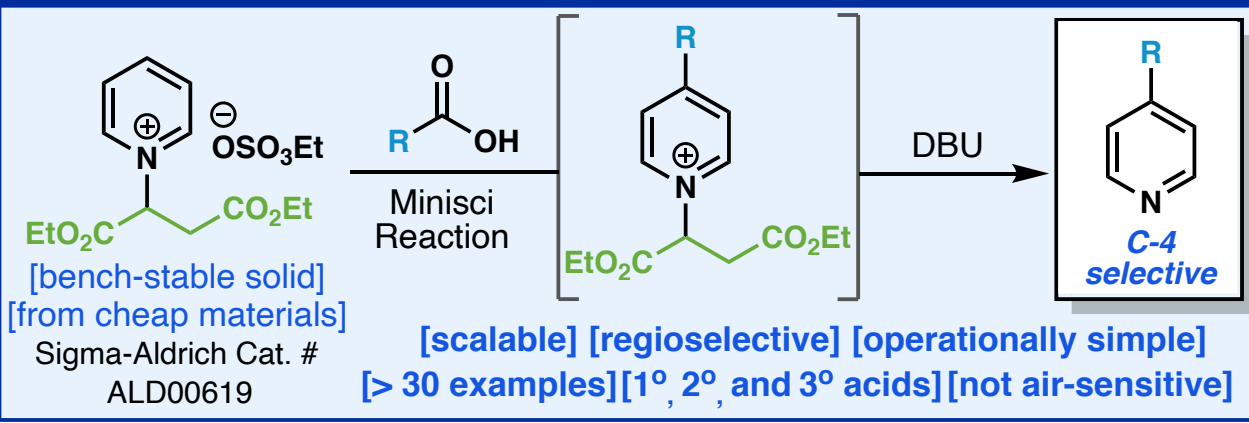

